\title{
On Some Elliptic Curves with Large Sha
}

\author{
Harvey E. Rose
}

\section{CONTENTS}

1. Introduction

2. Method

3. Rank-Zero Curves

4. Observations About the Calculations

References

Keywords: elliptic curve, rank

AMS Classification: Primary: 11G05, 11D28; Secondary: 14H52
We consider a class of elliptic curves many of whose associated Shafarevich-Tate groups $\amalg$ are relatively large, and give examples of curves with $\mathrm{o}(\amalg)=\mathrm{k}^{2}$ for all $k \leq 100$.

\section{INTRODUCTION}

Let $p$ be a prime satisfying $p \equiv 1(\bmod 8)$ throughout, and let $C(n)$ denote the elliptic curve

$$
C(n): y^{2}=x^{3}+n x,
$$

where $n \in \mathbb{Z}$. We shall mainly be concerned with the case $n=p^{3}$. Further, for the curve $C(n)$, let $r(C(n))$ denote the (Mordell-Weil) rank over $\mathbb{Q}$, and $s t(C(n))$ denote the (analytic) order of the Shafarevich-Tate group $\amalg_{C(n)}$. We shall assume that the full Birch and Swinnerton-Dyer conjecture holds for all curves under consideration; see [Silverman 1986] for further details. The conjecture has been established in the rank zero case, except possibly for the 2 component of the formula; see [Rubin 1991].

Whilst undertaking some general investigations on the elliptic curves $C(n)$ for various small $n$, we noted that in the cases when $n=p^{3}$ a surprising number of the curves had comparatively large values for $s t(C(n))$; for instance $s t\left(C\left(233^{3}\right)\right)=64$ and $s t\left(C\left(433^{3}\right)\right)=81$. This phenomenon was also noted for the curves $C\left(2 p^{3}\right)$ but to a lesser extent. After some further computations it became clear that the curves $C\left(p^{3}\right)$ regularly have large sha; and hence it was possible, and thought to be worthwhile, to produce a list of elliptic curves with $o(\amalg)=k^{2}$ for each $k$ in some typical range. We chose $k \leq 100$ as being attainable in a few weeks using a reasonably fast machine, although the last entry found, for $k=98$, did extend this timetable somewhat (and so it is remarkable in this case that a second prime occurs so soon after the first; although there are a number of

(C) A K Peters, Ltd. 1058-6458/2000 \$0.50 per page Experimental Mathematics 9:1, page 85 
similar instances, for example when $k=6$ or 35 ). See Table 2.

Cassels [1964] showed that there are elliptic curves with arbitrarily large Shafarevich-Tate groups Ш by considering quadratic twists by many different primes. Recently de Weger [1998] has given some specific examples of curves with large sha, his largest satisfies $o(\amalg)=224^{2}$. He also discusses the Goldfeld-Szpiro Conjecture, first considered in [Goldfeld and Szpiro 1995], relating the size of $\amalg$ to the conductor; see Section 4E.

A prime $p$ is called a $G$-prime if it can be expressed in the form $p=x^{2}+64 y^{2}$ (or, equivalently, if 2 is a quartic residue modulo $p$ ). A easy extension of this gives: $p^{3}$ can be expressed in the form

$$
p^{3}=x_{1}^{2}+64 y_{1}^{2} \quad \text { with } \quad\left(x_{1}, y_{1}\right)=1
$$

if and only if $p$ is a $G$-prime. Repeating the argument given in [Silverman 1986, Chapter 10] for the curves $C(p)$, we see that $C\left(p^{3}\right)$ has rank zero or two provided we assume, as we are doing, that the Birch and Swinnerton-Dyer Conjecture holds. (Note. The curve $C\left(p^{3}\right)$ is a quadratic twist of $C(p)$.) In [Rose 1995] we showed, using elementary methods, that $r(C(p))=0$ if $p$ is not a $G$-prime (and so the conjecture is not needed in this case); an exactly similar argument shows that $r\left(C\left(p^{3}\right)\right)=0$ when $p$ is not a $G$-prime, and again the conjecture is only needed in the $G$-prime case.

\section{METHOD}

For $p \equiv 1(\bmod 8)$ consider the elliptic curve $C\left(p^{3}\right)$. Note first that, whilst the discriminant of this curve is $64 p^{9}$, its conductor is $64 p^{2}$, and so it is as easy to calculate the value of $L(s)$-function at $s=1$ for the curve $C\left(p^{3}\right)$ as it is for $C(p)$ (as these curves have the same conductor). The calculations were undertaken using the method given in [Buhler et al. 1985] and the computer package Pari/GP 1.39.

In [Rose 1997] we conjecture that the probability for the curve $C(p)$ to have rank 2 is $O\left(p^{-1 / 8}\right)$ (this is backed up with some numerical evidence and the implied constant is close to $3 / 2$ ). The computations undertaken for this paper suggest that a similar estimate applies for the curves $C\left(p^{3}\right)$; that is, the probability of the rank of $C\left(p^{3}\right)$ equalling two is $O\left(p^{-3 / 8}\right)$. The data given in Table 1 provides

\begin{tabular}{|rclllll|}
\hline 89 & 6529 & 26249 & 41177 & 52673 & 67057 & 83089 \\
601 & 8969 & 26417 & 43441 & 54401 & 67129 & $83177^{*}$ \\
937 & $12697^{*}$ & $26497^{*}$ & 43721 & 54497 & 70921 & 84857 \\
1889 & 13913 & 27449 & 45281 & 57073 & 71233 & 86161 \\
2969 & 14249 & 29569 & 47057 & 57529 & 71761 & 87641 \\
3257 & 16633 & 32009 & 47609 & 57697 & $73417^{*}$ & 88873 \\
3529 & 17881 & 32377 & 47713 & 60089 & 75289 & 91873 \\
3673 & $25057^{*}$ & 35449 & 49681 & 65729 & 77249 & 96001 \\
4289 & 25409 & 40577 & 52489 & 66569 & 79537 & 96137 \\
\hline
\end{tabular}

TABLE 1. Primes $p \equiv 1(\bmod 8)$ less than $10^{5}$ for which the curve $C\left(p^{3}\right)$ has rank 2. The asterisk means that $r(C(p))=0$.

some evidence for the validity of this estimate. It is perhaps also of interest to note that there is no close correspondence between the ranks of $C(p)$ and $C\left(p^{3}\right)$ for fixed $p$ - for many primes $p, C(p)$ has rank 2 and $C\left(p^{3}\right)$ has rank 0 , whilst those $p$ marked with an asterisk in Table 1 satisfy the opposite: namely, $r(C(p))=0$ and $r\left(C\left(p^{3}\right)\right)=2$. In the remaining cases in this table both curves have rank 2. Note also that, for all the asterisked primes $p$ in the table, we have $s t(C(p))=64$ using data given in [Rose 1997]; for larger $p$ this equation will probably need to be replaced by the condition $64 \mid s t(C(p))$. Note that $s t(C(p))$ need not be a power of two even in the rank 2 case, for example $s t(C(51137))=9$ as noted in [Rose 1997].

We have confirmed that these curves have rank 2 (by finding two independent generators) for the first three primes only, although one generator is known in 20 cases. In the remaining cases we are relying on the Birch and Swinnerton-Dyer conjecture, and the fact that our calculated estimate for the value of the $L(s)$-function at $s=1$ equals zero to an accuracy of at least four places. It would be a major undertaking to find the generators for the remaining curves; in no case will elementary (that is, quadratic) arguments help.

\section{RANK-ZERO CURVES}

We consider now the elliptic curves $C\left(p^{3}\right)$ with rank zero; note that in this case the Birch-SwinnertonDyer conjecture has been established except for the power of 2 in their formula; see [Rubin 1991]. We have calculated the values of the $L(s)$-functions of these curves at $s=1$ for all primes congruent to 
1 modulo 8 up to 150000 , and up to 230000 for $G$ primes congruent to 1 or 33 modulo 40 only; a summary of the results is given in Table 2. We curtailed the calculations once we had found at least one entry in every line of Table 2, further details are available from the author via e-mail. We also calculated these $L$-function values in two higher, randomly chosen, ranges: 1200000 to 1205000 , and 4100100 to 4105100. All calculations were performed to an accuracy of at least three decimal places; this was sufficient to give, using the Birch and Swinnerton-Dyer conjecture, the value of $s t\left(C\left(p^{3}\right)\right)$ as this number is a square integer $k^{2}$ whose parity can be determined in advance, see Section $4 \mathrm{C}$ below. Also we found that the larger the value of $s t\left(C\left(p^{3}\right)\right)$ the better was the accuracy of the calculation. Typical examples of actual calculated values are:

$$
\begin{aligned}
& \operatorname{st}\left(C\left(229321^{3}\right)\right)=8464.0733 \approx 8464=92^{2}, \\
& \operatorname{st}\left(C\left(219361^{3}\right)\right)=2.8927 \approx 4
\end{aligned}
$$

(here 219361 is a $G$-prime, so the $s t$ value is an even square).

\section{OBSERVATIONS ABOUT THE CALCULATIONS}

\section{A. The Spread of Values of $k$}

All values of $k$ occur and, generally speaking, they occur with a similar frequency. It seems reasonable to assume that for all $k$ there are infinitely many primes $p$ such that

$$
\operatorname{st}\left(C\left(p^{3}\right)\right)=k^{2},
$$

although the frequency of these occurrences probably drops considerably as $p$ increases. For example the values $k=1,2$ or 3 do not occur in the range $1200000<p<1205000$, the smallest value of $s t\left(C\left(p^{3}\right)\right)$ for rank zero curves in this range is 16 .

Further the first prime $p$ for which the displayed equation above holds increases relatively smoothly with $k$, except that there is a slight tendency for this prime to be larger than 'normal' when $k$ has the form $k=2 n$ and $n$ is odd. Examples are when $k=6,26,50$ and 98 . This is probably not significant; for instance, although the smallest prime $p$ with $\operatorname{st}\left(C\left(p^{3}\right)\right)=2500$ is $p=79769$, there are at least eleven further primes with this property less then 200000. Finally note that there is also a tendency for the 'first' prime to be congruent to $3 \bmod 5$ (or, to a lesser extent, congruent to $1 \bmod 5$ ); this is also probably not significant but explains the choice of primes between 150001 and 230000 above.

\section{B. The Size of Values $k$}

Compared with some previously published tables, for example Cremona [1997], the sizes of the Shafarevich-Tate groups for the curves under consideration are relatively large. We have if $p<50000$ the largest value for $\operatorname{st}\left(C\left(p^{3}\right)\right)$ is 7744 , for the prime 46681 ; if $p<10^{5}$ the largest value is 11025 , for the prime 99233 ; if $p<150000$ the largest value is $28561=169^{2}$, for the prime 137873 .

Further in the range $1200000<p<1205000$ the largest $s t$ value is $111556=334^{2}$ for the prime 1200833 , and in the range $4100100<p<4105100$ we found the values

$$
\begin{aligned}
& \operatorname{st}\left(C\left(4102393^{3}\right)\right)=391^{2}, \\
& \operatorname{st}\left(C\left(4103353^{3}\right)\right)=474^{2}, \\
& \operatorname{st}\left(C\left(4105033^{3}\right)\right)=635^{2}=403225,
\end{aligned}
$$

which is the largest explicitly calculated value of sha for any elliptic curve known to the author.

\section{C. G and Non-G Primes}

For the curves $C\left(p^{3}\right)$,

$\operatorname{st}\left(C\left(p^{3}\right)\right)$ is even if and only if $p$ is a $G$-prime.

We used this to complete the table below by considering only $G$-primes between 150000 and 230000 . Note that, for the curves $C(p)$, we have $4 \mid s t(C(p))$ for all $p$ and

$$
16 \mid \operatorname{st}(C(p)) \text { if and only if } p \text { is a } G \text {-prime; }
$$

see [Rose 1995]. Also note that although $C\left(p^{3}\right)$ is a quadratic twist of $C(p)$ there is no precise relationship between their corresponding 'shas'. For example $s t(C(56081))=6^{2}$ whilst $s t\left(C\left(56081^{3}\right)\right)=55^{2}$.

\section{D. Relationship Between $C\left(p^{3}\right)$ and $C(p)$ for G-Primes $p$}

There is some connection between the 2-component of $s t\left(C\left(p^{3}\right)\right)$ and the rank of $C(p)$. Using the data given in [Rose 1995; 1997], the following properties hold for $p<10^{5}$ for the curves under consideration:

(a) If $4 \| s t\left(C\left(p^{3}\right)\right)$ then $r(C(p))=0$.

(b) If $16 \mid s t\left(C\left(p^{3}\right)\right)$ then either $r(C(p))=2$, or $r(C(p))=0$ and $64 \mid s t(C(p))$. 


\begin{tabular}{|c|c|c|c|c|c|c|c|c|c|c|c|c|c|c|c|c|c|c|c|}
\hline$k$ & $n$ & $p_{1}$ & $p_{2}$ & $k$ & $n$ & $p_{1}$ & $p_{2}$ & $k$ & $n$ & $p_{1}$ & $p_{2}$ & $k$ & & $p_{1}$ & $p_{2}$ & $k$ & & $p_{1}$ & $p_{2}$ \\
\hline 1 & 96 & 17 & 41 & 22 & 43 & 3761 & 7841 & 43 & 16 & 310 & & 64 & & & & 85 & & 49433 & \\
\hline 2 & 68 & 257 & 577 & 3 & 33 & 2753 & & 44 & 15 & & & 65 & & & & 86 & & & \\
\hline 3 & 116 & 137 & 241 & 4 & 45 & 3313 & & 45 & 16 & & & 66 & & & & 8 & 2 & & \\
\hline 4 & 126 & 7 & & 25 & 46 & 2953 & 456 & 46 & 5 & & & 67 & & & & 8 & 1 & & 93 \\
\hline 5 & 123 & 313 & 401 & 26 & 25 & 19433 & 2629 & 47 & 7 & & & 68 & & & & 8 & 2 & & \\
\hline 6 & 72 & 2833 & 2857 & 27 & 27 & & & 48 & 13 & & & 69 & & & & 90 & 0 & & \\
\hline 7 & 82 & 641 & 2417 & 28 & 34 & 116 & & 49 & 8 & & & 70 & & & & 91 & 2 & & \\
\hline 8 & 98 & 233 & 1153 & 29 & 22 & & & 50 & 4 & & & 71 & $I$ & & & 92 & 0 & & \\
\hline 9 & 92 & 433 & & 30 & 32 & 9281 & & 51 & 7 & & & 72 & 3 & & & 93 & 2 & & \\
\hline 10 & 60 & 1721 & 2441 & 1 & 19 & 124 & & 52 & 13 & & & 73 & 2 & & & 94 & 0 & & \\
\hline 11 & 63 & 953 & 2713 & 32 & 31 & & & 53 & 7 & & & 74 & 1 & & & 95 & 0 & & \\
\hline 12 & 91 & 1753 & 01 & 33 & 20 & 8513 & & 54 & 1 & & & 75 & 2 & & & 96 & & & \\
\hline 13 & 70 & 21 & 50 & 34 & 7 & 219 & & 55 & 3 & & & 76 & 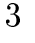 & & & 97 & 2 & & \\
\hline 14 & 50 & 01 & 37 & 35 & 25 & & & 56 & 5 & & & 77 & & & & 98 & 0 & 93 & 224993 \\
\hline 15 & 70 & 49 & 114 & 36 & 32 & & & 57 & 8 & & & 78 & & & & 99 & 0 & 21 & 13 ? \\
\hline 16 & 60 & 93 & & 37 & 19 & 15473 & & 58 & 4 & & & 79 & & 43913 & 146 & 100 & 1 & 153 & 103553 \\
\hline 17 & 49 & 81 & & 38 & 10 & 28001 & & 59 & 2 & & & 80 & & & & 101 & 1 & 92033 & \\
\hline 18 & 36 & 817 & 12497 & 39 & 17 & 17401 & 197 & 60 & 10 & 236 & & 81 & & 82193 & 940 & 102 & 0 & 114073 & 201673 \\
\hline 19 & 42 & 93 & & 40 & 14 & 24953 & & 61 & 3 & & & 82 & & 87281 & 1239 & 103 & 0 & & \\
\hline 20 & 60 & 2273 & 361 & 41 & 13 & 7193 & 121 & 62 & 2 & & & 83 & & 23593 & & 104 & 0 & 109433 & 117881 \\
\hline 21 & 37 & 4793 & 6329 & 42 & 12 & 25913 & 32993 & 63 & 5 & 35593 & 49033 & 84 & & 68713 & 109313 & 105 & 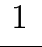 & 99233 & \\
\hline
\end{tabular}

TABLE 2. For each $k \leq 105$, the second column gives the number $n$ of primes $p<10^{5}$ for which $s t\left(C\left(p^{3}\right)\right)=k^{2}$. The columns headed $p_{1}$ and $p_{2}$ give the two smallest primes $p$ for which $s t\left(C\left(p^{3}\right)\right)=k^{2}$; only one such prime is known for $k=101,103$ and 105 .

In this final case, divisibility cannot be replaced by equality: for example if $p=50177$, we have $r(C(p))=0$ whilst $s t(C(p))=256$.

\section{E. The Goldfeld-Szpiro Conjecture}

In [Goldfeld and Szpiro 1995] it was conjectured that elliptic curves defined over $\mathbb{Q}$ with Shafarevich-Tate group $\amalg$, conductor $N$, and $\varepsilon>0$, satisfy

$$
o(\amalg) \ll N^{1 / 2+\varepsilon} .
$$

Let GS denote the ratio $o(\amalg) / \sqrt{N}$, and dW denote the ratio $o(\mathrm{~W}) / \Delta^{1 / 12}$ where $\Delta$ is the discriminant of the curve in question. In [de Weger 1998] there are several examples of elliptic curves with GS larger than 1, the largest value being 6.893 for the curve mentioned in the introduction. In the same article de Weger proves, assuming the validity of the Birch and Swinnerton-Dyer Conjecture in the rank zero case, that there are many elliptic curves with dW larger than unity (the precise statement is: for all $\varepsilon>0$, there exist infinitely many elliptic curves $E$ defined over $\mathbb{Q}$ with the property $\left.o\left(\amalg_{E}\right) \gg \Delta^{1 / 12-\varepsilon}\right)$. For the curves discussed in this paper all values of GS are less than 0.040 but some satisfy $\mathrm{dW}>1$. The six curves $C\left(p^{3}\right)$ with the largest values of GS are:

\begin{tabular}{rccc}
\multicolumn{1}{c}{$p$} & GS & dW & $s t\left(C\left(p^{3}\right)\right)$ \\
\hline 23593 & 0.0365 & 2.559 & 6889 \\
16553 & 0.0349 & 2.241 & 4624 \\
233 & 0.0343 & 0.759 & 64 \\
7193 & 0.0292 & 1.522 & 1681 \\
11353 & 0.0286 & 1.672 & 2601 \\
73 & 0.0274 & 0.453 & 16 \\
\hline
\end{tabular}

Incidently, the elliptic curve $C\left(4105033^{3}\right)$, having the largest sha we have found to date (see Section $4 \mathrm{~B}$ above), has $\mathrm{GS}=0.01228$ and $\mathrm{dW}=3.1264$.

\section{REFERENCES}

[Buhler et al. 1985] J. P. Buhler, B. H. Gross, and D. B. Zagier, "On the conjecture of Birch and SwinnertonDyer for an elliptic curve of rank 3", Math. Comp. 44:170 (1985), 473-481.

[Cassels 1964] J. W. S. Cassels, "Arithmetic on curves of genus 1, VI: The Tate-Šafarevič group can be arbitrarily large", J. Reine Angew. Math. 214/215 (1964), 65-70. 
[Cremona 1997] J. E. Cremona, Algorithms for modular elliptic curves, 2nd ed., Cambridge Univ. Press, Cambridge, 1997.

[Goldfeld and Szpiro 1995] D. Goldfeld and L. Szpiro, "Bounds for the order of the Tate-Shafarevich group", Compositio Math. 97:1-2 (1995), 71-87. Special issue in honour of Frans Oort.

[Rose 1995] H. E. Rose, "On a class of elliptic curves with rank at most two", Math. Comp. 64:211 (1995), 1251-1265, S27-S34.
[Rose 1997] H. E. Rose, "On some classes of elliptic curves with rank two or three", preprint, 1997.

[Rubin 1991] K. Rubin, "The "main conjectures" of Iwasawa theory for imaginary quadratic fields", Invent. Math. 103:1 (1991), 25-68.

[Silverman 1986] J. H. Silverman, The arithmetic of elliptic curves, vol. 106, Graduate Texts in Math., Springer, New York, 1986.

[de Weger 1998] B. M. M. de Weger, " $A+B=C$ and big Ш's", Quart. J. Math. Oxford Ser. (2) 49:193 (1998), 105-128.

Harvey E. Rose, Department of Mathematics, University Walk, Bristol, BS8 1TW, United Kingdom (h.e.rose@bris.ac.uk)

Received May 26, 1998; accepted in revised form March 8, 1999 